\title{
RESEARCH
}

Open Access

\section{Built and social environmental factors influencing healthy behaviours in older Chinese immigrants to Australia: a qualitative study}

Ester Cerin ${ }^{1,2,3^{*}}$ (D), Andrea Nathan ${ }^{4}$, Wing Ka Choi ${ }^{1}$, Winsfred Ngan ${ }^{5}$, Shiyuan Yin ${ }^{5}$, Lukar Thornton ${ }^{5}$ and Anthony Barnett ${ }^{1}$

\begin{abstract}
Background: Neighbourhood environments influence older adults' health and health-enhancing behaviours, such as physical activity, eating a healthy diet and socialising. However, little is known about the effects of the neighbourhood environment on the health of older immigrants, the number of which is rapidly increasing in developed countries. Using Nominal Group Technique (NGT) sessions, this study of older Chinese immigrants to urban Melbourne, Australia, examined built and social environmental facilitators of and barriers to regular engagement in physical activity, eating a healthy diet and regular contact with other people.

Methods: Participants were recruited from four types of neighbourhoods stratified by walkability and proportion of Chinese dwellers. Twelve NGTs, four specific to each of physical activity, healthy diet and social contacts were conducted in Mandarin or Cantonese (91 participants). NGT responses from groups addressing the same questions were aggregated, similar items were combined, and scores combined across groups. Inductive thematic analysis was used to categorise answers into higher-order themes of factors associated with each behaviour.

Results: For physical activity, 29 facilitators and 28 barriers were generated with the highest ranked facilitator and barrier being "proximity to destinations" and "poor/inadequate public transport", respectively. For healthy diet, 25 facilitators and 25 barriers were generated, the highest ranked facilitator and barrier were "high food safety standards/regulations" and "lack of family/household members' social support for a healthy diet". The social contacts NGTs generated 23 facilitators and 22 barriers, with the highest ranked facilitator and barrier being "proximity to destinations and activities" and "poor public transport", respectively.

Discussion: Independent living arrangements and the accessibility of destinations of daily living (e.g., bilingual health services, libraries, places of worship and grocery stores / supermarkets), recreational facilities, affordable public transport, and community centres and activities for Chinese people are key elements for promoting regular engagement in physical activity, healthy eating and socialising in older Chinese immigrants. Governments should plan for the provision of this basic infrastructure of community facilities for older immigrants.
\end{abstract}

Keywords: Older adults, Chinese immigrants, Neighbourhood environment, Physical activity, Walkability, Diet, Social contacts, Transport, Destinations

\footnotetext{
*Correspondence: Ester.Cerin@acu.edu.au

${ }^{1}$ Mary Mackillop Institute for Health Research, Australian Catholic University,

Melbourne, Australia

${ }^{2}$ School of Public Health, The University of Hong Kong, Hong Kong, Hong

Kong SAR, China

Full list of author information is available at the end of the article
}

(c) The Author(s). 2019 Open Access This article is distributed under the terms of the Creative Commons Attribution 4.0 International License (http://creativecommons.org/licenses/by/4.0/), which permits unrestricted use, distribution, and reproduction in any medium, provided you give appropriate credit to the original author(s) and the source, provide a link to the Creative Commons license, and indicate if changes were made. The Creative Commons Public Domain Dedication waiver (http://creativecommons.org/publicdomain/zero/1.0/) applies to the data made available in this article, unless otherwise stated. 


\section{Background}

The neighbourhood environment is an important source of influences on older adults' health [1-5] and healthenhancing behaviours, including participation in physical activity [6-8], eating a healthy diet $[9,10]$ and socialising $[11,12]$. For example, older people living in neighbourhoods with better access to daily destinations and amenities have reported higher levels of participation in active transport [7], physical activity [6] and social activities $[11,13]$. Neighbourhood environments are particularly relevant to older adults because they experience more limited mobility and are, therefore, more reliant on facilities near to where they live [4].

Studies of neighbourhood effects on health-enhancing behaviours have mainly focused on the general population of older adults living in specific areas, while there is a dearth of information on these issues in relation to older immigrants $[14,15]$. Older immigrants are particularly susceptible to being impacted by their neighbourhood environment due to cultural differences and poor language proficiency after migration, which limit their mobility [16]. For this reason and the fact that the number of older immigrants to developed countries is rapidly increasing (e.g., increase of $>11$ million from 1990 to 2017) [17], it is important to identify neighbourhood characteristics that influence health-enhancing behaviours of older immigrants in these regions. Oceania (specifically, Australia and New Zealand) is the developed region with the largest proportion of, and increase in, older immigrants in the world [17]. According to 2016 census data, Chinese immigrants are the largest nonEnglish-speaking minority group in Australia [18], accounting for $2.7 \%$ of Australia's total population and $1.6 \%$ of the total ageing (65+ year old) population [19]. As most immigrants to Australia are settling in capital cities, it is important to investigate characteristics of the neighbourhood environment that might impact on health-enhancing behaviours of older Chinese immigrants to urban Australia. This information can be used to help identify groups of immigrants that are particularly vulnerable to various health-related problems arising from dramatic environmental shifts (e.g., change in available foods, opportunities for physical activity and social contacts) and identify interventions that may mitigate these negative effects.

The aim of the present study was to identify built and social environmental facilitators of and barriers to regular engagement in physical activity, eating a healthy diet and regular contact with other people among older Chinese immigrants to urban Australia. We focused on Melbourne metropolitan area because Melbourne was the capital city with the largest population growth in 2016-17, with overseas migration accounting for $64.1 \%$ of the growth [18]. Given the dearth of information on the issues covered by the present study, a qualitative approach was implemented. Qualitative studies are key to informing intervention strategies that are effective and sustainable. This is because they enable the identification of high-priority problems and approaches that are feasible and acceptable to the target population. Qualitative research is particularly important for the planning and implementation of interventions for non-mainstream, hard-to-reach, isolated subgroups of the population (e.g., older Chinese immigrants to Western countries) because they are understudied and usually require approaches that differ from those that are effective in the mainstream population [20]. According to empirical evidence, intervention strategies tailored to minority groups that are based on findings from qualitative formative research are more effective than those that do not collect or utilise such information [21]. For the purpose of this study, a qualitative, structured, brainstorming technique (i.e., Nominal Group Technique, NGT) was adopted [22]. Compared to other qualitative techniques, such as focus groups, NGT sessions have been shown to produce a greater number of diverse ideas and facilitate balanced participation between group members [22].

\section{Methods}

A qualitative study of older Chinese immigrants was conducted to identify built and social environmental facilitators and barriers to healthy behaviours. Ethics approval was granted by the Deakin University Human Ethics Advisory Group, Faculty of Health (reference number 161-2014).

\section{Participants}

A purposive convenience sample of older Chinese immigrants was recruited from four types of neighbourhoods varying in walkability and proportion of Chinese dwellers. This approach ensured the recruitment of a heterogeneous sample of participants exposed to different levels of access to services, availability of Chinese ethnic foods and opportunities to socialise with residents of Chinese background.

Neighbourhoods in metropolitan Melbourne (defined as Statistical Areas Level 1, SA1; the smallest area of output for the Australian Census of Population and Housing) were identified and stratified as being high or low walkable using a transport-related walkability index in Geographic Information Systems (GIS) including information on dwelling density, street intersection density and land use mix. Low and high walkable neighbourhoods were those with a walkability index falling in the first and fourth quartiles, respectively. This strategy has been often employed in studies on the neighbourhood environment to maximise variation in types of environments [23-25]. Census data were used to classify 
neighbourhoods into those with a medium percentage of Chinese dwellers $(5-10 \%$ of residents were Chinese) or high percentage of Chinese dwellers $(>10 \%$ of residents were Chinese). Thus, four categories of neighbourhoods were created: high walkable and high \% Chinese (HW/ $\mathrm{HC})$; high walkable and medium \% Chinese (HW/MC); low walkable and high \% Chinese (LW/HC); and low walkable and medium \% Chinese (LW/MC).

Participants were then recruited using a variety of approaches, such as advertisements in Chinese newspapers, delivering presentations at Chinese seniors' groups and Chinese speaking churches, and posters in community libraries and medical centres. Presentations were delivered in Cantonese, Mandarin or English, depending on the audience preferences. All printed recruitment material was prepared in Traditional Chinese, Simplified Chinese and English. Potential participants contacted project staff speaking their preferred language (Cantonese, Mandarin or English) to assess eligibility status. Participants were eligible if they were ethnically Chinese and not born in Australia; aged 60 years and over; able to walk unassisted; able to speak and read Chinese (Cantonese/ Mandarin) and/or English; able to attend a data collection session lasting up to $1.5 \mathrm{~h}$; did not suffer from a diagnosed medical condition that affects cognitive functioning (e.g., dementia or major depression); and lived in pre-selected neighbourhoods. Participants were recruited between October and November 2014. If eligible, then written consent was provided.

\section{Data collection}

Participants completed a 15 item Demographic and Health questionnaire, which was returned along with a signed consent form. NGT sessions were held from October 2014 to March 2015. NGT is a well-established structured, multistep, 'brainstorming' technique used to generate and prioritise responses from a group of participants to a carefully articulated question intended to address specific information needs [22]. Overall 12 NGT sessions were held, four sessions specific to each of three healthy behaviours: physical activity (defined as 'any type of activity that uses your muscles and uses more energy than just resting'), healthy diet (defined as 'eating a wide variety of different foods including plenty of fruits and vegetables, nuts, whole grains, beans and peas, lean meats, fish and chicken; and eating foods that are fatty, salty or sweet sparingly'), and social contacts (defined as 'regular pleasant contacts with other people in the place where you live, here in Australia, such as friends, family members, colleagues, neighbours, acquaintances or people who work in your local area (e.g., shop assistant)). The NGT sessions involved a total of 91 participants, ranging from five to 13 participants per session. Each participant was randomly assigned to and took part in only one NGT session on a particular healthy behaviour. NGT sessions were held at Deakin University or at a local community centre and conducted in either Mandarin or Cantonese (the participants' preferred languages). The NGT sessions lasted 60-90 min.

Three research assistants were trained to facilitate sessions. All sessions followed the same structure and procedures adopted in previous NGT studies seeking to identify built and social environmental factors associated with healthy behaviours [24, 26, 27]. Participants were told that the purpose of the NGT was to provide ideas on what they thought could be changed in living environments to create better and healthier places for elderly Chinese to live in. First, participants were asked to "list things about places where Chinese elderly live that make it easy to be physically active, eat healthy, or have contacts with people". Participants were told that it could be about people in those places, buildings, facilities, services or infrastructure. They then wrote down ideas on a piece of paper without discussing them with other participants. Once the response generation process was completed, the facilitator asked participants to nominate the responses written on their own worksheet using a round-robin approach until all ideas were exhausted. All responses were written on a whiteboard for the participants to read. Generated items were reviewed and clarified if ambiguous or combined if equivalent. Once finalised, each participant anonymously selected and wrote down the three most relevant items on a blank card and ranked them by importance using scores from 1 to 3 (3 denoting the most important item). In the second part of the NGT session participants were asked to "list aspects of places where Chinese elderly live that make it difficult to be physically activity, eat healthy, or have contacts with people". The same round-robin listing and voting steps then occurred. For the third and final part, participants were asked "if they could change one thing about the place they live in that would help them exercise more, have a healthy diet, or have contacts with people, what would it be?". No round-robin listing or voting occurred for this third part. The main purpose of the third part of the data collection was to identify potential interventions that would have local relevance and compare those with the more general list of facilitators / barriers to health-enhancing behaviours identified by the same participants.

\section{Analysis}

Differences in socio-demographic and health-related characteristics between behaviour-specific NGT groups were tested using chi-squared tests. All NGT responses were entered in a database. The responses from NGT groups addressing the same questions about a specific healthy behaviour were aggregated. Specifically, items 
that were very similar were first combined to create a new list of distinctive (non-overlapping) items. The total score for each distinctive item was computed by summing the scores it received from each participant (possible individual participant's scores ranged from 0 to 3 ). In the present study, the minimum score that an item could receive was 0 . This could occur if no participants deemed the specific item to be one of the three most relevant generated items. The maximal score that an item could receive was equal to the total number of participants taking part in one of the four behaviourspecific NGTs multiplied by 3 (e.g., a total of 32 participants participating in physical-activity-focused NGTs could yield a maximum item score of 96). This could occur if all participants deemed the specific item to be the most relevant in the list and gave it a score of 3 . Inductive thematic analysis was used to identify and further categorise items into higher-order themes of factors associated with each behaviour (e.g., the environmental physical activity facilitators "park nearby" and "cycling track nearby" were categorised under the theme "proximity to destinations"). Three investigators reviewed these aggregations, discussed differences in opinions and resolved disagreements. Scores for specific themes within each healthy behaviour (e.g., proximity to destinations as facilitators of engagement in physical activity) were then computed by aggregating (i.e., summing up) the scores on the relevant items.

\section{Results}

\section{Participant characteristics}

Overall, 91 older Chinese immigrants participated in the 12 groups held: four NGTs on physical activity, healthy diet, and social contacts, respectively. Sociodemographic and health-related characteristics are presented in Table 1 stratified by NGT healthy behaviour. Participant ages ranged from 60 to 85 years $(M=71.13$, $\mathrm{SD}=5.91$ ). Across all participants, duration of residency in Australia ranged from 1 to 58 years $(M=12.12, S D=$ 12.41). Most participants lived with their adult children. The distributions of participants by neighbourhood walkability $(p=0.640)$, neighbourhood-level percentage of Chinese dwellers $(p=0.775)$, sex $(p=0.851)$, age groups $(p=0.220)$, ethnicity $(p=0.739)$, language spoken at home $(p=0.483)$, residency in Australia $(p=0.733)$, living arrangements $(p=0.904)$, self-reported health $(p=$ $0.627)$ and number of chronic conditions $(p=0.317)$ did not differ across the three behaviour-specific NGTs.

\section{Environmental factors related to physical activity}

A total of 57 responses (29 facilitators and 28 barriers) were generated. The aggregated responses ranked as important across all four NGT groups are presented in Table 2, while more detailed sub- categories of responses, including those that no participant considered among the three most important, can be found in Additional file 1: Table S1. The highest ranked facilitator of being physically active was proximity to destinations, endorsed by three out of four NGT groups (including both groups of participants living in low walkable areas). Specifically, participants reported that living close to parks, recreational facilities, and shopping centres made it easy to be physically active. Having access to destinations for physical activity (without specifying how far away from home they were located) was the next highest ranked environmental facilitator. Next was the importance of having access to organised social groups and structured and diverse activities to participate in, which was the only facilitator endorsed by all four group types. Other responses endorsed by more than one NGT group included aspects of the home environment supporting physical activity (particularly important to participants from low walkable areas); social support for physical activity (endorsed by two out of four group types, both of which were living in high walkable areas); and pet ownership.

The highest ranked barrier to being physically active was poor/inadequate public transport, which was the only response endorsed by all four NGT groups. Other environmental barriers to physical activity endorsed by more than one NGT group included: language barriers; lack of destinations supporting physical activities (important to residents of low walkable areas); having limited social groups/activities to join (endorsed by the two high walkable NGT groups); individual health-related and socio-economic factors (endorsed by participants living in high walkable areas); lack of information about community activities; and aspects of the home environment not conducive to physical activity (endorsed by the two groups living in low walkable areas).

When asked "if you could change one thing about your place you live in that would help you engage in more physical activity, what would it be?", the most popular responses were (in order): access to and improvement of Chinese community centres with facilities for recreational physical activity; improving living arrangements; proximity of destinations for recreational physical activity; and reducing language barriers. Each of these items was raised by participants from at least two NGT groups (Table 3 and Additional file 1: Table S2).

\section{Environmental factors related to a healthy diet}

Overall, 25 facilitators and 25 barriers (50 in total) were generated. Table 4 outlines the aggregated categories of facilitators and barriers to following a healthy diet across all four NGTs, while Additional file 1: Table S3 also reports sub-categories of barriers and facilitators. 
Table 1 Participant socio-demographic and health-related characteristics by NGT topic

\begin{tabular}{|c|c|c|c|c|c|c|c|c|c|}
\hline & & \multicolumn{2}{|c|}{$\begin{array}{l}\text { Physical activity } \\
(n=37)\end{array}$} & \multicolumn{2}{|c|}{$\begin{array}{l}\text { Healthy diet } \\
(n=27)\end{array}$} & \multicolumn{2}{|c|}{$\begin{array}{l}\text { Social contacts } \\
(n=27)\end{array}$} & \multicolumn{2}{|c|}{$\begin{array}{l}\text { Total } \\
(n=91)\end{array}$} \\
\hline & & $n$ & $\%$ & $n$ & $\%$ & $\mathrm{n}$ & $\%$ & $\mathrm{n}$ & $\%$ \\
\hline \multirow[t]{2}{*}{ Neighbourhood walkability } & High & 18 & 48.6 & 16 & 59.3 & 13 & 48.1 & 47 & 51.7 \\
\hline & Low & 19 & 51.4 & 11 & 40.7 & 14 & 51.9 & 44 & 48.3 \\
\hline \multirow[t]{2}{*}{ Neighbourhood \% Chinese } & Medium & 16 & 43.2 & 14 & 51.9 & 12 & 44.4 & 42 & 46.1 \\
\hline & High & 21 & 56.8 & 13 & 48.1 & 15 & 55.6 & 49 & 53.9 \\
\hline \multirow[t]{2}{*}{ Sex } & Male & 14 & 37.8 & 9 & 33.3 & 11 & 40.7 & 34 & 37.4 \\
\hline & Female & 23 & 62.2 & 18 & 66.7 & 16 & 59.3 & 57 & 62.6 \\
\hline \multirow[t]{5}{*}{ Age group } & 60-64 years & 11 & 29.7 & 5 & 18.5 & 1 & 3.7 & 17 & 18.7 \\
\hline & $65-69$ years & 6 & 16.2 & 8 & 29.6 & 8 & 29.6 & 22 & 24.2 \\
\hline & $70-74$ years & 6 & 16.2 & 6 & 22.2 & 10 & 37.0 & 22 & 24.2 \\
\hline & 75-79 years & 11 & 29.7 & 6 & 22.2 & 6 & 22.2 & 23 & 25.3 \\
\hline & $80+$ years & 3 & 8.1 & 2 & 7.4 & 2 & 7.4 & 7 & 7.7 \\
\hline \multirow[t]{7}{*}{ Place of origin } & Shanghai province & 10 & 27.0 & 8 & 29.6 & 4 & 14.8 & 22 & 24.2 \\
\hline & Guangdong province & 6 & 16.2 & 3 & 11.1 & 6 & 22.2 & 15 & 16.5 \\
\hline & Beijing province & 4 & 10.8 & 3 & 11.1 & 4 & 14.8 & 11 & 12.1 \\
\hline & Other provinces ${ }^{a}$ & 12 & 32.4 & 6 & 22.2 & 6 & 22.2 & 24 & 26.4 \\
\hline & Hong Kong SAR & 4 & 10.8 & 3 & 11.1 & 6 & 22.2 & 13 & 14.3 \\
\hline & Taiwan & 0 & 0.0 & 1 & 3.7 & 0 & 0.0 & 1 & 1.1 \\
\hline & Other $^{b}$ & 1 & 2.7 & 3 & 11.1 & 1 & 3.7 & 5 & 5.5 \\
\hline \multirow[t]{3}{*}{ Language spoken at home } & Mandarin only & 20 & 54.1 & 14 & 51.9 & 13 & 48.1 & 47 & 51.6 \\
\hline & Cantonese only & 7 & 18.9 & 8 & 29.6 & 11 & 40.7 & 26 & 28.6 \\
\hline & Other $^{c}$ & 10 & 27.0 & 5 & 18.5 & 3 & 11.1 & 18 & 19.8 \\
\hline \multirow[t]{4}{*}{ Residency in Australia } & $<5$ years & 20 & 54.1 & 10 & 37.0 & 11 & 40.7 & 41 & 45.1 \\
\hline & $5-14$ years & 6 & 16.2 & 6 & 22.2 & 6 & 22.2 & 18 & 19.8 \\
\hline & $15-24$ years & 5 & 13.5 & 7 & 25.9 & 4 & 14.8 & 16 & 17.6 \\
\hline & $25+$ years & 6 & 16.2 & 4 & 14.8 & 6 & 22.2 & 16 & 17.6 \\
\hline \multirow[t]{3}{*}{ Living arrangements } & Living alone & 2 & 5.4 & 2 & 7.4 & 3 & 11.1 & 7 & 7.7 \\
\hline & Living with others but not adult children & 14 & 37.8 & 10 & 37.0 & 8 & 29.6 & 32 & 35.2 \\
\hline & Living with adult children & 21 & 56.8 & 15 & 55.6 & 16 & 59.3 & 52 & 57.1 \\
\hline \multirow[t]{5}{*}{ Self-rated health } & Poor & 1 & 2.7 & 0 & 0.0 & 3 & 11.1 & 4 & 4.4 \\
\hline & Fair & 15 & 40.5 & 11 & 40.7 & 10 & 37.0 & 36 & 39.6 \\
\hline & Good & 12 & 32.4 & 12 & 44.4 & 8 & 29.6 & 32 & 35.2 \\
\hline & Very good & 8 & 21.6 & 4 & 14.8 & 6 & 22.2 & 18 & 19.8 \\
\hline & Excellent & 1 & 2.7 & 0 & 0.0 & 0 & 0.0 & 1 & 1.1 \\
\hline \multirow[t]{3}{*}{ Number of chronic health conditions } & None & 9 & 24.3 & 10 & 37.0 & 4 & 14.8 & 23 & 25.3 \\
\hline & One & 11 & 29.7 & 8 & 29.6 & 7 & 25.9 & 26 & 28.6 \\
\hline & Two or more & 17 & 45.9 & 9 & 33.3 & 16 & 59.3 & 42 & 46.2 \\
\hline
\end{tabular}

${ }^{\mathrm{a}}$ Other includes Chongqing, Sichuang, Anhui, Fujian, Jiangsu, Shandong, Zhejiang, Heilongjiang, Liaoning, Shanxi, Tianjin provinces. ${ }^{\mathrm{b}}$ Other includes Malaysia, Vietnam. ${ }^{\circ}$ Other includes English, Chiu Chau dialect, Shang Hai dialect, Southern Fujian dialect, Vietnamese, Taiwanese or any combination of these with Mandarin and/or Cantonese

The highest ranked facilitator, which was endorsed by all four NGT groups, was having high food safety standards/regulations. This was followed by the provision of educational information on healthy eating through various outlets (Chinese newspapers, community talks), which was endorsed by three out of four NGT groups. Other frequently-endorsed facilitators to eating a healthy diet included availability of healthy foods in grocery stores and having family/household social support for a healthy diet. 
Table 2 Built and social environmental facilitators and barriers to engagement in physical activity

\begin{tabular}{|c|c|c|}
\hline Categories of responses generated during NGT sessions & $\begin{array}{l}\text { Total votes (across } \\
\text { groups) }\end{array}$ & $\begin{array}{l}\text { Group types endorsing } \\
\text { response }\end{array}$ \\
\hline \multicolumn{3}{|l|}{ Facilitators } \\
\hline Proximity to destinations & 63 & LW/HC, LW/MC, HW/HC \\
\hline Easy access to destinations for physical activity (regardless of distance) & 34 & $\mathrm{HW} / \mathrm{MC}, \mathrm{LW} / \mathrm{HC}$ \\
\hline Access to social group/activities & 28 & $\begin{array}{l}\mathrm{HW} / \mathrm{MC}, \mathrm{LW} / \mathrm{HC}, \mathrm{LW} / \mathrm{MC} \text {, } \\
\mathrm{HW} / \mathrm{HC}\end{array}$ \\
\hline Information available on health and community events, initiatives or services in Chinese & 18 & HW/MC \\
\hline Home environment providing opportunities for physical activity & 17 & LW/HC, LW/MC, HW/HC \\
\hline Social support for physical activity & 14 & $\mathrm{HW} / \mathrm{MC}, \mathrm{HW} / \mathrm{HC}$ \\
\hline $\begin{array}{l}\text { Enjoying household physical activity [characteristic describing Chinese older residents } \\
\text { in the community] }\end{array}$ & 14 & $\mathrm{HW} / \mathrm{HC}$ \\
\hline Opportunities to facilitate integration in the community & 13 & HW/MC \\
\hline Quality public transport & 8 & HW/MC \\
\hline Pet ownership & 5 & LW/MC, HW/HC \\
\hline Safety from traffic & 5 & LW/MC \\
\hline Caring responsibilities & 2 & $\mathrm{HW} / \mathrm{HC}$ \\
\hline Living near other Chinese elders & 1 & LW/MC \\
\hline \multicolumn{3}{|l|}{ Barriers } \\
\hline Poor/inadequate public transport & 40 & $\begin{array}{l}\mathrm{HW} / \mathrm{MC}, \mathrm{LW} / \mathrm{HC}, \mathrm{LW} / \mathrm{MC} \text {, } \\
\mathrm{HW} / \mathrm{HC}\end{array}$ \\
\hline Language barriers & 36 & $\mathrm{HW} / \mathrm{MC}, \mathrm{LW} / \mathrm{HC}$ \\
\hline Lack of destinations/facilities supporting physical activity & 34 & LW/HC, LW/MC \\
\hline Limited social group/activities for Chinese people & 29 & $\mathrm{HW} / \mathrm{MC}, \mathrm{HW} / \mathrm{HC}$ \\
\hline $\begin{array}{l}\text { Health-related and socio-economic factors [characteristics describing Chinese older residents } \\
\text { in the community] }\end{array}$ & 28 & $\mathrm{HW} / \mathrm{MC}, \mathrm{HW} / \mathrm{HC}$ \\
\hline Lack of information on community activities & 23 & $\mathrm{HW} / \mathrm{MC}, \mathrm{LW} / \mathrm{HC}$ \\
\hline Home environment not conducive to physical activity & 20 & LW/HC, LW/MC \\
\hline Lack of social support for physical activity & 9 & LW/HC \\
\hline Lack of public housing supporting independence & 3 & HW/MC \\
\hline
\end{tabular}

${ }^{\mathrm{a}}$ Maximum of 6 votes per participant (3-2-1 scoring). ${ }^{\mathrm{b}} \mathrm{HW}=$ High Walkability; LW = Low Walkability; MC = Medium \% Chinese; HC = High \% Chinese

Endorsed by all NGT groups, the highest ranked barrier to healthy eating was to do with a lack of family/ household support for healthy food options. Other barriers endorsed by more than one NGT group were: financial barriers to purchasing healthy foods (endorsed by residents of high walkable areas); high availability or prevalence of unhealthy food options available in food outlets (endorsed by residents of high walkable areas); distance to grocery stores (endorsed by respondents living in areas with medium percentage of Chinese residents); and cultural preferences for an unhealthy diet and unhealthy cooking practices (endorsed by both NGT groups living in high walkable areas).

When asked to identify one aspect to change that would help to follow a healthy diet, the most popular responses were having better provision of educational information on healthy eating and better access to grocery stores and/or fresh produce (mentioned by three NGT groups) (Table 3 and Additional file 1: Table S4). Other aspects mentioned by more than one NGT group were improved access to and/or management of Chinese grocery stores, improved public transport; and reduction of unhealthy food options in food outlets.

\section{Environmental factors related to social contacts}

The total number of responses generated was 45 (23 facilitators and 22 barriers). Table 5 presents the aggregated categories of facilitators and barriers to having contact with people across all four NGTs. Subcategories of facilitators and barriers are detailed in Additional file 1: Table S5.

The highest ranked facilitator to social contact was proximity to destinations and activities, endorsed by all NGT groups. Specific destinations mentioned by participants included shops (especially Chinese stores), library, recreational facilities, GPs, restaurants, and community 
Table 3 One thing that Chinese older immigrants would change in their own community to help them be more physically active, eat a healthy diet and have contacts with people

\begin{tabular}{|c|c|c|}
\hline Categories of responses & $\begin{array}{l}\text { Total votes (across } \\
\text { groups) }\end{array}$ & $\begin{array}{l}\text { Group types endorsing } \\
\text { response }^{\mathrm{a}}\end{array}$ \\
\hline \multicolumn{3}{|l|}{ Physical activity } \\
\hline $\begin{array}{l}\text { Access to and improvement of Chinese elderly community centres with facilities for } \\
\text { recreational physical activity }\end{array}$ & 13 & $\begin{array}{l}\text { LW/HC, LW/MC, HW/HC, } \\
\text { HW/MC }\end{array}$ \\
\hline Improving living arrangements & 12 & HW/MC, LW/HC \\
\hline Proximity to destinations for recreational physical activity & 5 & HW/MC, LW/MC \\
\hline Reducing language barriers & 4 & HW/MC, LW/HC \\
\hline Improvements in the neighbourhood environment & 1 & $\mathrm{HW} / \mathrm{MC}$ \\
\hline Reducing financial barriers & 1 & HW/HC \\
\hline Opportunities to facilitate integration & 1 & $\mathrm{HW} / \mathrm{MC}$ \\
\hline \multicolumn{3}{|l|}{ Healthy diet } \\
\hline Better provision of educational information on healthy eating in the community & 7 & LW/HC, LW/MC, HW/MC \\
\hline Better access to grocery stores and/or fresh produce & 7 & HW/MC, LW/HC, HW/HC \\
\hline Improve access to and/or management of Chinese grocery stores & 4 & LW/MC, HW/HC \\
\hline Improved public transport & 4 & HW/MC, LW/MC \\
\hline Reduce unhealthy food options in food outlets & 2 & $\mathrm{LW} / \mathrm{HC}, \mathrm{HW} / \mathrm{HC}$ \\
\hline Interventions aimed at improving diet-related habits & 2 & HW/HC \\
\hline Financial independence & 1 & HW/MC \\
\hline \multicolumn{3}{|l|}{ Social contacts } \\
\hline Improving public transport & 7 & HW/MC, LW/HC, LW/MC \\
\hline Improving access to places for social/group activities for Chinese elderly & 7 & LW/HC, HW/MC \\
\hline Increasing the number of activities in the community for Chinese elderly & 3 & HW/HC \\
\hline Provision of opportunities for the improvement of English language skills & 2 & HW/MC, LW/MC \\
\hline Provision of services in Chinese & 2 & LW/MC \\
\hline Living near other Chinese people & 2 & LW/MC, HW/MC \\
\hline Promoting neighbourliness & 1 & LW/HC \\
\hline Housing design to facilitate social contacts & 1 & LW/MC \\
\hline Instrumental support for mobility & 1 & LW/MC \\
\hline Improved access to telecommunication & 1 & LW/MC \\
\hline
\end{tabular}

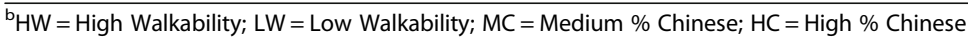

centres. Endorsed by both NGT groups living in high walkable areas, the next highest facilitator to social contact was the availability of community services and media in Chinese. This was followed by the following facilitator categories endorsed by at least two NGT groups: opportunities to learn English (endorsed by both groups living in areas with a medium percentage of Chinese residents); access to destinations and activities (endorsed by three of four NGT groups); good public transport; and living near or with Chinese people (endorsed by both NGT groups living in low walkable communities).

Two barriers to maintaining contact with people were ranked highly and endorsed by all four NGT groups. These were poor public transport, particularly its inconvenience and infrequency, and language barriers. Limited or poor access to destinations and social group/activities for Chinese people was also a highly ranked barrier to social contacts, which was endorsed by three out of four NGT groups.

The most popular changes to make in the community that would help older Chinese to have contacts with people were: improving access to places for social/group activities for Chinese elderly; improving public transport; and increasing the number of activities in the communities for Chinese elderly (Table 3 and Additional file 1: Table S6). The first two types of changes were endorsed by at least two NGT groups.

\section{Discussion}

Although population ageing, urbanisation and international migration to developed countries are three main 
Table 4 Built and social environmental facilitators and barriers to a healthy diet

\begin{tabular}{|c|c|c|}
\hline Categories of responses generated during NGT sessions & $\begin{array}{l}\text { Total votes (across } \\
\text { groups) }\end{array}$ & $\begin{array}{l}\text { Group types endorsing } \\
\text { response }\end{array}$ \\
\hline \multicolumn{3}{|l|}{ Facilitators $^{c}$} \\
\hline High food safety standards/regulations & 59 & $\begin{array}{l}\text { HW/MC, LW/HC, LW/ } \\
\text { MC, HW/HC }\end{array}$ \\
\hline Providing educational information on healthy eating in the community & 44 & $\begin{array}{l}\mathrm{HW} / \mathrm{MC}, \mathrm{LW} / \mathrm{HC}, \mathrm{LW} / \\
\mathrm{MC}\end{array}$ \\
\hline Family/household members social support for a healthy diet & 22 & LW/MC, HW/HC \\
\hline Availability of healthy foods in grocery stores & 20 & $\begin{array}{l}\mathrm{HW} / \mathrm{MC}, \mathrm{HW} / \mathrm{HC}, \mathrm{LW} / \\
\mathrm{HC}\end{array}$ \\
\hline Food nutritional labelling in Chinese & 13 & $\begin{array}{l}\text { LW/MC, LW/HC, HW/ } \\
\text { HC }\end{array}$ \\
\hline $\begin{array}{l}\text { Preference for a healthy diet and healthy cooking practices [characteristic describing Chinese older } \\
\text { residents in the community] }\end{array}$ & 9 & LW/MC, HW/HC \\
\hline Proximity to Chinese grocery store & 7 & LW/MC \\
\hline \multicolumn{3}{|l|}{ Barriers } \\
\hline Lack of family/household members social support for a healthy diet & 54 & $\begin{array}{l}\text { HW/MC, LW/HC, LW/ } \\
\text { MC, HW/HC }\end{array}$ \\
\hline Financial barriers to purchasing healthy foods & 26 & $\mathrm{HW} / \mathrm{MC}, \mathrm{HW} / \mathrm{HC}$ \\
\hline High availability or prevalence of unhealthy food options available in food outlets & 23 & $\mathrm{HW} / \mathrm{MC}, \mathrm{HW} / \mathrm{HC}$ \\
\hline $\begin{array}{l}\text { Cultural preference for an unhealthy diet and unhealthy cooking practices [characteristic describing } \\
\text { Chinese older residents in the community] }\end{array}$ & 19 & $\begin{array}{l}\text { LW/MC, LW/HC, HW/ } \\
\text { HC }\end{array}$ \\
\hline Distance to grocery store & 14 & HW/MC, LW/MC \\
\hline Limited availability of fresh vegetables & 9 & $\mathrm{HW} / \mathrm{HC}$ \\
\hline Poor adherence to food safety standards/regulations & 6 & $\mathrm{HW} / \mathrm{HC}$ \\
\hline Unable to grow fruit and vegetables at home & 4 & $\mathrm{HW} / \mathrm{MC}$ \\
\hline Language barriers to reading food nutritional labels & 4 & LW/MC \\
\hline Poor public transport (low frequency) & 2 & LW/MC \\
\hline Misleading or inaccurate educational information on healthy eating & & LW/MC \\
\hline
\end{tabular}

${ }^{\mathrm{a}}$ Maximum of 6 votes per participant (3-2-1 scoring). ${ }^{\mathrm{b}} \mathrm{HW}=$ High Walkability; LW = Low Walkability; MC = Medium \% Chinese; HC = High \% Chinese. ${ }^{\mathrm{c} A d d i t i o n a l}$ votes $=12$ additional votes for 'Facilitators' due to scored items listing two factors being counted twice under different response categories

global demographic trends [28], there is a general dearth of information on how the urban environment of a host country impacts on the well-being of older immigrants. This qualitative study aimed to address this knowledge gap by identifying built and social environmental facilitators and barriers to three health-enhancing behaviours in older Chinese immigrants to Melbourne, Australia's second largest city.

\section{Facilitators and barriers to engagement in physical activity}

Regular engagement in physical activity is associated with a plethora of health benefits including risk reduction of premature death [29] and major chronic diseases [30]. Mid- and older-aged East Asian immigrants (mainly Chinese) to Australia have been found to be less physically active than non-immigrants [31], although Chinese older adults residing in their country of origin exhibit much higher levels of physical activity than older Australians [32-35]. These differences in activity levels have been in part attributed to better accessibility to destinations and public transport in Chinese than Australian cities [32, 36]. The results from the present study reiterate the importance of proximity and access to destinations as key facilitators to engagement in physical activity for older Chinese immigrants residing in both low- and high-walkable neighbourhoods relative to the local context of Melbourne, Australia. Also, the lack of destinations was identified as a major barrier to physical activity only by those living in low walkable areas typified by low levels of mixed-use buildings.

Types of destinations considered to be particularly important were parks, walking tracks, shops, recreational centres and community centres. Except for community centres, all these types of destinations have been associated with higher levels of physical activity among older adults in general [6-8]. Community centres appear to be particularly important to older immigrants. In this study, access to Chinese elderly community centres (e.g., Chinese seniors club) with facilities for physical activity was 
Table 5 Built and social environmental facilitators and barriers to social contacts

\begin{tabular}{|c|c|c|}
\hline Categories of responses generated during NGT sessions & $\begin{array}{l}\text { Total votes (across } \\
\text { groups) }\end{array}$ & $\begin{array}{l}\text { Group types endorsing } \\
\text { response }\end{array}$ \\
\hline \multicolumn{3}{|l|}{$\overline{\text { Facilitators }^{c}}$} \\
\hline Proximity to destinations and activities & 72 & $\begin{array}{l}\text { LW/HC, LW/MC, HW/HC, } \\
\text { HW/MC }\end{array}$ \\
\hline Availability of community services and media in Chinese & 24 & $\mathrm{HW} / \mathrm{MC}, \mathrm{HW} / \mathrm{HC}, \mathrm{LW} / \mathrm{MC}$ \\
\hline Access to destinations and activities & 19 & HW/MC, LW/MC, HW/HC \\
\hline Opportunities to learn English & 19 & HW/MC, LW/MC \\
\hline Good public transport & 18 & LW/MC, HW/HC \\
\hline Living near or with Chinese people & 10 & LW/HC, LW/MC \\
\hline Unlimited access to telecommunication & 2 & LW/MC \\
\hline \multicolumn{3}{|l|}{ Barriers } \\
\hline Poor public transport & 66 & $\begin{array}{l}\text { HW/MC, LW/HC, LW/MC, } \\
\text { HW/HC }\end{array}$ \\
\hline Language barriers & 49 & $\begin{array}{l}\text { HW/MC, LW/HC, LW/MC, } \\
\text { HW/HC }\end{array}$ \\
\hline $\begin{array}{l}\text { Limited or poor access to destinations and social group/activities for Chinese people (or } \\
\text { residents) }\end{array}$ & 41 & $\mathrm{HW} / \mathrm{HC}, \mathrm{HW} / \mathrm{MC}, \mathrm{LW} / \mathrm{MC}$ \\
\hline Living separately from other Chinese people (public housing arrangements) & 5 & LW/MC \\
\hline Cultural differences limiting integration & 4 & $\mathrm{HW} / \mathrm{HC}$ \\
\hline $\begin{array}{l}\text { Health-related and personality factors [characteristics describing Chinese older residents in } \\
\text { the community] }\end{array}$ & 3 & HW/MC \\
\hline
\end{tabular}

${ }^{a}$ Maximum of 6 votes per participant (3-2-1 scoring). ${ }^{\mathrm{b}} \mathrm{HW}=$ High Walkability; LW = Low Walkability; MC = Medium \% Chinese; HC = High \% Chinese. ${ }^{\mathrm{c} A d d i t i o n a l}$ votes $=1$ additional votes for 'Facilitators' and 6 for 'Barriers' due to scored items listing two factors being counted twice under different response categories

the most frequently endorsed single change in the community that participants thought would help them be more physically active. Previous studies have indicated that ethnic-specific community organisations are important sources of activities for older immigrants that, apart from offering affordable opportunities to engage in a particular activity (e.g., exercise), help them overcome social isolation and problems associated with language barriers by providing access to people of the same ethnic minority [37-39]. In connection with this issue, the present study found that participants from all four types of communities reported access to group (physical) activities as a major facilitator, and lack of group activities as a major barrier, to being physically active. It appears that the ability to link physical activities with local Chinese-speaking social networks is an important consideration for the promotion of physical activity in Chinese older immigrants [37].

As noted above, mirroring findings from other studies on older Chinese immigrants [38], this study suggests that factors related to language barriers and ways to mitigate them were deemed to influence the ability to engage in physical activity. Apart from access to group activities involving other Chinese-speaking residents, language-related facilitators/barriers identified in this study included the provision/lack of information on activities in Chinese, networking opportunities for English and Chinese older adults, and the (in) ability to communicate in English. According to Ip and colleagues [38], the lack of English language proficiency and the difficulties in accessing language support services, limit Chinese older adults' mobility and, hence, ability to find and engage in community activities, including leisure-time physical activity appropriate for their age. Furthermore, inadequate and infrequent public transport services were the most frequently reported mobility-related barriers to engagement in physical activity by all NGT groups. Given that the majority of older Chinese immigrants do not drive [37, 38], their participation in activities outside their immediate neighbourhood is dependent on their family members (e.g., children driving them to/from destinations) and/or access to public transport. The latter emerged as a particularly important factor in the present study, likely due to Chinese older immigrants preferring not to rely on their children for transportation because they see them as being 'always busy at work' [37, 38].

Household environment characteristics (e.g., having a garden or exercise equipment at home) and individuallevel characteristics of Chinese older immigrants (e.g., enjoying household activities, health problems, financial hardship, lack of time and pet ownership) were less frequently reported as factors impacting on engagement in physical activity. Most of these factors have previously been identified as physical activity barriers or facilitators 
in general populations of adults and older adults [4042]. Among these factors, financial problems are particularly relevant to older immigrants who tend to be financially dependent on their children because they are less likely to accumulate adequate resources for independent living and often have reduced government pensions and benefits due to their immigrant status [37, 43]. As a result of these financial constraints, their choice of activities in the community are limited.

Linked to financial independence are living arrangements. Older Chinese immigrants often live with their adult children and children-in-law due to economic constraints, language barriers and cultural reasons [37, 38, $44,45]$. However, most of them express a desire for independent living arrangements, i.e., for living in a household separate from their children [37, 45]. Typically, the main reasons behind this desire are poor-quality relationships at home $[38,45,46]$, seeking more autonomy in lifestyle and ability to fulfil one's own needs [37, 38]. This explains why, in the present study, the provision of independent living arrangements was considered one of the main single changes that would help older Chinese immigrants be more physically active. Specifically, participants maintained that independent living would provide them the freedom and time to engage in leisuretime physical activity. Also, they indicated that public housing for Chinese elderly would facilitate participation in group activities and improve their mood and desire to participate in such activities.

\section{Facilitators and barriers to a healthy diet}

While mid-aged and older East Asian (including Chinese) immigrants to Australia have been found to report lower fruit and vegetable intake than their Australian counterparts [31], Osypuk and colleagues [47] reported that Chinese who lived in immigrant enclaves had healthier diets that individuals who did not. Further, the diets of ethnic minorities who migrate to Western countries are usually worse than when living in their country of origin [48]. These findings suggest that healthy eating among older Chinese immigrants may be determined by the physical and social attributes of their home and neighbourhood environments. However, these issues remain understudied.

In this study, having high food safety standards was by far the most frequently endorsed facilitator to healthy eating. Food safety in China has been and is remaining a major concern [49]. In this regard, Alcorn and Ouyang [50] reported that Chinese residents considered food safety the second greatest risk they faced in daily life (after earthquakes). It is, thus, perhaps not surprising that older Chinese immigrants, most of whom had migrated to Australia from China in recent times $(<5$ years), would consider high food safety standards and regulations an essential factor for healthy eating. However, they did not identify low food safety standards as barriers to healthy eating, nor did they select improvements in food safety standards as the single thing they would change in their host community to help them follow a healthier diet. These patterns of findings are likely reflective of their confidence in Australian food safety standards which are higher than those in China [51].

In this study, the provision of educational information on healthy eating was the second most frequently endorsed facilitator to healthy eating and one of the two most popular single changes older Chinese immigrants thought would help them adopt or sustain a healthy diet. Participants emphasized the need for information in Chinese that would help them address language barriers. They noted the utility of having information on healthy eating published in the local Chinese newspapers and/or delivered through talks and workshops (in Chinese) organised by local community groups and/or health services.

Improvements in some eating habits (e.g., decreased use of deep-frying, increased fruit and vegetable intake) as a result of improved nutritional knowledge have been previously observed in Chinese immigrants to Western countries [52-54]. These findings are in line with research on mainstream populations. In fact, nutritional knowledge has been consistently identified as one of the most important psychosocial determinants of a healthy diet. For example, in their systematic review, Guillaumie and colleagues [55] noted that over half of the studies that examined the effects of knowledge on fruit and/or vegetable intake reported positive effects. Also, a recent study found that knowledge was a strong predictor of vegetable intake in East Asians [56].

Two strong psychosocial determinants of healthy eating in mainstream populations that emerged as key facilitators/barriers in this study are social influences $[55,56]$ and eating/cooking habits [55]. As noted earlier, a large proportion of older Chinese immigrants live with, and depend on the financial support of, their adult children $[37,43]$. These constrained living arrangements limit their freedom to choose the foods they eat. In this study, living with family members (mainly children) who prefer unhealthy fast foods was by far the most prevalent perceived barrier to healthy eating. It has been suggested that younger Chinese immigrants tend to more easily adopt a Western diet, while older Chinese immigrants prefer a healthier traditional diet [54]. However, their ability to follow their dietary preferences depends on their financial independence and status in the household [37]. In this regard, it is notable that, in the present study, financial constraints were the second most highly ranked perceived barrier to healthy eating, further highlighting the disadvantaged socio-economic position 
of older Chinese immigrants. In line with these findings are those of mainstream studies that have identified healthy food affordability as a consistent determinant of healthy food choices and purchasing behaviours being particularly important to lowincome older adults [57, 58].

This study identified comparative availability of healthy and unhealthy foods in the community and grocery stores / supermarkets as an important factor impacting on healthy eating. Specifically, the availability of fresh produce and affordable fruit and vegetables in supermarkets were considered facilitators of healthy eating by participants living in different types of neighbourhoods. On the other hand, the availability of unhealthy foods in supermarkets and restaurants, and the limited choice of fresh produce in grocery stores were identified as barriers among those living in high walkable areas. The same group of participants indicated that an increase in amounts and varieties of fruit and vegetables in grocery stores and supermarkets would help them follow a healthy diet. Residents of high walkable areas usually have relatively good access to supermarkets and shops [7]. Thus, for them, availability of healthy foods rather than access to outlets that sell such foods may be a more salient determinant of healthy eating. Previous studies have noted that the comparative availability of healthy and unhealthy options in food outlets played a key role in residents' dietary patterns [58]. This was particularly the case among low-income [59] and ethnic minority groups [60], with the latter reporting difficulties in obtaining traditional ethnic-specific fresh produce.

Similarly to that observed in mainstream populations [58], older Chinese immigrants indicated that accessibility of healthy foods in the community - namely, distance to grocery stores - influenced their diet. Having access to grocery stores (Chinese or generic), markets and good public transport to/from grocery stores were among the single changes they would introduce in the local community to help them eat healthily. Lack of transportation to access grocery stores and long distances to grocery stores were aspects of the neighbourhood environment that emerged as important barriers to healthy eating in other studies on socially disadvantaged older adults [57] and other age groups [58].

\section{Facilitators and barriers to social contacts}

Socially isolated individuals are at increased risk of dying prematurely [61] and not recovering from life threatening events [62]. Social isolation and feelings of loneliness are also associated with depression [63], mental and cognitive health decline [64], and poorer sleep [65] and quality of life [46]. Older Chinese immigrants to Western countries are particularly vulnerable to social isolation because of their older age, relocation to an unfamiliar environment, insufficient language skills to build new networks, lack of information and access to services, and cultural barriers [66, 67]. They frequently report insufficient quantity of social relationships outside their household [45] which, in conjunction with language and cultural barriers, leads to them feeling lonely [46]. It is, thus, important to identify ways to promote and enhance social contacts in this population.

Three main sources of influences on the older Chinese immigrants' ability to socialise emerged in the present study. These were access and availability of appropriate places and activities to meet people, language barriers and transportation. Proximity and access to destinations and activities were the facilitators most frequently endorsed by all NGT groups. Also, improvements in these community characteristics were identified as key interventions that would help older Chinese immigrants have contact with other people. In particular, participants emphasised the importance of having access to activities and destinations for Chinese residents, including Chinese community centres, senior clubs, libraries, churches and health services. Proximity to shops and restaurants was also found to be a positive contributor to social activities. Previous studies on older Chinese immigrants found that Chinese community centres and services were vital sources of social networks [38, 39, 45, 68]. Makwarimba and colleagues [68] noted that older immigrants want to have their own ethnic senior centres where they can meet people who speak their own language, cook their ethnic food and access service information through dissemination activities. This is a particularly important issue for older Chinese immigrants co-residing with their adult children, lacking private space for socialising at home, and experiencing strained relationships with their younger household members $[37,38,46]$ that result in lower levels of psychological well-being [69].

Having access to destinations and activities for Chinese people was one of the ways in which participants could address their lack of proficiency in the English language as a barrier to socialising. Other ways were living near other Chinese people and having opportunities to learn English. In this regard, it was suggested that the local community and government should provide English language classes for older immigrants and public housing for Chinese people. In an earlier study of older Chinese immigrants to Australia, Ip and colleagues [38] found that one of the consequences of poor English language skills was that older immigrants did not have the confidence to venture out on their own to visit their friends or attend social activities, which reinforced their social isolation.

Finally, in this study, access to good public transport was identified as a facilitator and poor public transport 
as the most important barrier to having social contacts. Participants living in communities differing in level of walkability and prevalence of Chinese residents all perceived public transport inconvenience, unaffordability and unintelligibility (e.g., public transport stop signs and bus numbers being unclear) as a major barrier. Similar findings were observed in other studies on older Chinese in Australia [38] and Canada [45].

\section{Study limitations}

This study has several limitations. Firstly, NGT procedures do not allow participants to elaborate on the reasons for selecting specific facilitators or barriers to health-enhancing behaviours. NGTs were conducted with a non-probabilistic, purposively selected sample of older Chinese immigrants to one Australian city. Hence, the findings are not generalizable to all older Chinese immigrant groups in Australia and in other countries. The characteristics of the built and social environment in other Australian cities and countries are likely to differ $[70,71]$ and impact on the perceived factors influencing participation in physical and social activities as well as the ability to follow a healthy diet. Also, the findings may not apply to other ethnic groups. Future work should include larger and more representative samples of older Chinese from diverse geographical locations.

\section{Practical implications}

Despite the limitations of this study, the findings have useful practical implications. The household and community environments are particularly important to older Chinese immigrants because they determine their level of independence and access to healthy foods, affordable transportation, opportunities for physical and social activities, and social networks that provide information and support. The provision of public housing in walkable neighbourhoods with access to destinations for daily living, recreational facilities and affordable public transport would enable older Chinese immigrants to live independently from their adult children and, as a result, follow a healthier diet and have more time for engaging in physical and social activities with other older Chinese residents. Destinations for daily living that should be provided within walking distance from residential buildings are Chinese or bilingual health services, libraries, places of worship, and grocery stores / supermarkets selling affordable ethnic-specific fresh produce. It would be particularly important to establish a dedicated community centre for older Chinese that would provide a place for physical and social activities and be a dissemination point for information about community events, health and government policies. Local communities should also offer multi-purpose programs, such as English classes that promote physical activity and healthy eating, which have been shown to be effective in minority groups [72].

The present study confirms the utility of qualitative research in identifying problems (e.g., isolation) and solutions (e.g., English classes that promote healthy eating) related to minority groups' healthy behaviours that do not typically apply, or do not apply to the same extent, to the mainstream population. The findings from this and future similar qualitative investigations can be used to inform the planning and implementation of tailored interventions aimed at promoting a physically and socially active lifestyle and healthy eating in older Chinese immigrants to Western countries. Within the context of Melbourne, the current study suggests that effective and sustainable interventions would need to focus on environmental changes that improve the independence and decrease the level of isolation of older Chinese immigrants. The types of interventions identified by study participants (e.g., the provision of public housing, affordable public transport and ethnic-specific fresh produce) would require the coordinated engagement of state and local transportation, urban planning, health and human services departments in consultation with representatives of the community. In order to respond to community needs, policymakers and practitioners ought to be informed of the existence of these needs and possible ways to meet them. The provision of research summaries from this and similar qualitative studies that also contain policy recommendations may facilitate research translation and community-level action [73].

\section{Conclusions}

This study examined physical and social environmental facilitators and barriers to physical activity, healthy eating and social contacts in older Chinese immigrants to Melbourne, Australia. In line with previous investigations conducted in other Western, English-speaking geographical locations, the present study suggests that independent living arrangements and the accessibility of destinations of daily living, recreational facilities, affordable public transport and community centres and activities for Chinese people are key elements for promoting a healthy lifestyle and well-being in older Chinese immigrants. As immigration to developed countries is likely to continue in the future, governments should plan for the provision of this basic infrastructure of community facilities for older immigrants.

\section{Additional file}

Additional file 1: Table S1. Built and social environmental facilitators and barriers to physical activity. Table S2. One thing that Chinese older immigrants would change in their own community to help them be more physically active. Table S3. Built and social environmental 
facilitators and barriers to eating a healthy diet. Table S4. One thing that Chinese older immigrants would change in their own community to help them follow a healthy diet. Table S5. Built and social environmental facilitators and barriers to social contacts. Table S6. One thing that Chinese older immigrants would change in their own community to help them have contacts with people.

\section{Abbreviations}

HC: High neighbourhood proportion of Chinese dwellers; HW: High walkable; LC: Low neighbourhood proportion of Chinese dwellers; LW: Low walkable; NGT: Nominal Group Technique

\section{Acknowledgements}

We thank local walking groups, community centres and Chinese senior clubs for their assistance with participant recruitment.

\section{Authors' contributions}

EC designed the study, wrote the grant proposal, conceptualised the data analysis plan, analysed the data and wrote the manuscript. AN assisted with the data analysis and drafted parts of the method and results sections. WKC, WN and SY recruited the participants, prepared data collection material and ran the NGT sessions. LT assisted with the design of the study and selection of communities for participant recruitment. $A B$ assisted with the design of the study and writing of the manuscript. All authors critically commented on drafts of the manuscript and approved the the final version.

\section{Funding}

Seed funding - School of Exercise and Nutrition Sciences, Deakin University (2014).

\section{Availability of data and materials}

Available on request.

\section{Ethics approval and consent to participate}

Ethics approval was granted by the Deakin University Human Ethics Advisory Group, Faculty of Health (reference number HEAG-H 161-2014). Written informed consent for participation was obtained from participants.

\section{Consent for publication}

Not applicable.

\section{Competing interests}

The authors declare that they have no competing interests.

\section{Author details}

${ }^{1}$ Mary MacKillop Institute for Health Research, Australian Catholic University, Melbourne, Australia. ${ }^{2}$ School of Public Health, The University of Hong Kong, Hong Kong, Hong Kong SAR, China. ${ }^{3}$ Baker Heart and Diabetes Institute, Melbourne, Australia. ${ }^{4}$ Cancer Council Victoria, Melbourne, Australia. ${ }^{5}$ School of Exercise and Nutrition Science, Deakin University, Geelong, Australia.

\section{Received: 13 August 2019 Accepted: 14 November 2019}

Published online: 29 November 2019

\section{References}

1. Barnett A, Zhang CJP, Johnston JM, Cerin E. Relationships between the neighborhood environment and depression in older adults: a systematic review and meta-analysis. Int Psychogeriatr. 2018 Aug;30(8):1153-76. https:// doi.org/10.1017/S104161021700271X.

2. Besser LM, McDonald NC, Song Y, Kukull WA, Rodriguez DA. Neighborhood environment and cognition in older adults: a systematic review. Am J Prev Med. 2017;53(2):241-51. https://doi.org/10.1016/j.amepre.2017.02.013.

3. Paquet C, Coffee NT, Haren MT. Food environment, walkability, and public open spaces are associated with incident development of cardio-metabolic risk factors in a biomedical cohort. Health Place. 2014;28:173-6. https://doi. org/10.1016/j.healthplace.2014.05.001.

4. Rachele JN, Sugiyama T, Davies S, Loh VHY, Turrell G, Carver A, et al. Neighbourhood built environment and physical function among mid-toolder aged adults: a systematic review. Health Place. 2019:58:102137. https:// doi.org/10.1016/j.healthplace.2019.05.015.
5. Tani Y, Suzuki N, Fujiwara T, Hanazato M, Kondo N, Miyaguni Y, et al. Neighborhood food environment and mortality among older Japanese adults: results from the JAGES cohort study. Int J Behav Nutr Phys Act. 2018; 15(1):101. https://doi.org/10.1186/s12966-018-0732-y.

6. Barnett DW, Barnett A, Nathan A, Van Cauwenberg J, Cerin E, Council on Environment and Physical Activity (CEPA) - Older Adults working group. Built environmental correlates of older adults' total physical activity and walking: a systematic review and meta-analysis. Int J Behav Nutr Phys Act. 2017;14(1):103. https://doi.org/10.1186/s12966-017-0558-z.

7. Cerin E, Nathan A, van Cauwenberg J, Barnett DW, Barnett A, Council on Environment and Physical Activity (CEPA) - Older Adults working group. The neighbourhood physical environment and active travel in older adults: a systematic review and meta-analysis. Int J Behav Nutr Phys Act. 2017;14(1): 15. https://doi.org/10.1186/s12966-017-0471-5.

8. Van Cauwenberg J, Nathan A, Barnett A, Barnett DW, Cerin E. Council on environment and physical activity (CEPA)-older adults working group. Relationships between Neighbourhood physical environmental attributes and older Adults' leisure-time physical activity: a systematic review and meta-analysis. Sports Med. 2018:48(7):1635-60. https://doi.org/10.1007/s40279-018-0917-1.

9. Mercille G, Richard L, Gauvin L, Kestens Y, Shatenstein B, Daniel M, et al. Associations between residential food environment and dietary patterns in urban-dwelling older adults: results from the VoisiNuAge study. Public Health Nutr. 2012;15(11):2026-39. https://doi.org/10.1017/ S136898001200273X.

10. Sharkey JR, Johnson CM, Dean WR. Food access and perceptions of the community and household food environment as correlates of fruit and vegetable intake among rural seniors. BMC Geriatr. 2010;10:32. https://doi. org/10.1186/1471-2318-10-32.

11. Hand C, Law M, Hanna S, Elliott S, McColl MA. Neighbourhood influences on participation in activities among older adults with chronic health conditions. Health Place. 2012;18(4):869-76. https://doi.org/10.1016/j. healthplace.2012.03.004.

12. Schmidt T, Kerr J, Schipperijn J. Associations between Neighborhood Open Space Features and Walking and Social Interaction in Older Adults-A Mixed Methods Study. Geriatrics (Basel). 2019;4(3). https://doi.org/10.3390/ geriatrics4030041.

13. Bowling A, Stafford M. How do objective and subjective assessments of neighbourhood influence social and physical functioning in older age? Findings from a British survey of ageing. Soc Sci Med. 2007;64:2533-49. https://doi.org/10.1016/j.socscimed.2007.03.009.

14. Bird S, Kurowski W, Feldman S, Browning C, Lau R, Radermacher H, et al. The influence of the built environment and other factors on the physical activity of older women from different ethnic communities. J Women Aging. 2009;21(1):33-47. https://doi.org/10.1080/08952840802633669.

15. Chung J, Seo JY, Lee J. Using the socioecological model to explore factors affecting health-seeking behaviours of older Korean immigrants. Int J Older People Nursing. 2018;13(2):e12179. https://doi.org/10.1111/opn.12179.

16. Lin X, Bryant C, Boldero J, Dow B. Psychological well-being of older Chinese immigrants living in Australia: a comparison with older Caucasians. Int Psychogeriatrics. 2016;28(10):1671-9. https://doi.org/10.1017/ S1041610216001010

17. United Nations Department of Economic and Social Affairs (UN DESA) and HelpAge International. Ageing, older persons, and the 2030 agenda for sustainable development. New York: UN DESA and HelpAge International; 2017.

18. Simon-Davies J. Population and migration statistics in Australia. Research papers series 2018-19. Canberra: Parliament of Australia, Department of Parliamentary Services; 2018. https://parlinfo.aph.gov.au/parllnfo/download/ library/prspub/6377182/upload_binary/6377182.pdf

19. Australian Bureau of Statistics (ABS), 2016 Census of population and housing, cultural diversity in Australia, 2016. Census article on ABS website. https://www.abs.gov.au/ausstats/abs@.nsf/Lookup/by\%20Subject/2071.0 2 016 Main\%20Features Cultural\%20Diversity\%20Data\%20Summary 30. Accessed 11 May 2019.

20. Strolla LO, Gans KM, Risica PM. Using qualitative and quantitative formative research to develop tailored nutrition intervention material for diverse lowincome audience. Health Educ Res. 2006;21(4):465-76. https://doi.org/10. 1093/her/cyh072.

21. Gans KM, Risica PM, Strolla LO, Fournier L, Kirtania U, Upegui D, Zhao J, George T, Acharyya S. Effectiveness of different methods for delivering tailored nutrition edulation to low income, ethnically diverse adults. Int J Behav Nutr Physic Act. 2009;6:4. https://doi.org/10.1186/1479-5868-6-24. 
22. Van de Ven AH, Delbecq AL. The effectiveness of nominal, Delphi, and interacting group decision making processes. Acad Manag J. 1974;17(4): 605-21. https://doi.org/10.5465/255641.

23. Cerin E, Suen YN, Barnett A, Huang WYJ, Mellecker RR. Validity of a scale of neighbourhood informal social control relevant to pre-schoolers' physical activity: a cross-sectional study. SSM - Population health. 2017;3:57-65.

24. Suen YN, Cerin E, Wu SL. Parental practices encouraging and discouraging physical activity in Hong Kong Chinese pre-schoolers. J Phys Act Health. 2015;12:361-9.

25. Zhang CJP, Barnett A, Johnston JM, Lai PC, Lee RSY, Sit CHP, et al. Objectively-measured neighbourhood attributes as correlates and moderators of quality of life in older adults with different living arrangements: the ALECS cross-sectional study. Int J Environ Res Public Health. 2019;16(5):876

26. Cerin E, O'Connor TM, Mendoza JA, Thompson DI, Lee RE, Hughes SO, Baranowski T. A child-centered scale of informal social control for Latino parents of preschool-age children: development and validation. Hisp J Behav Sci. 2015;37(4):541-59.

27. He G, Cerin E, Huang WY, Wong SH. Understanding neighbourhood environment related to Hong Kong children's physical activity: a qualitative study using nominal group technique. PLoS One. 2014;9(9):e106578.

28. Leeson GW. The growth, ageing and urbanisation of our world. J Popul Ageing. 2018;11:107. https://doi.org/10.1007/s12062-018-9225-7.

29. WHO. Global health risks: Mortality and burden of disease attributable to selected major risks. Geneva: WHO; 2009.

30. Vogel T, Brechat PH, Leprêtre PM, Kaltenbach G, Berthel M, Lonsdorfer J. Health benefits of physical activity in older patients: a review. Int J Clin Pract. 2009;63(2):303-20. https://doi.org/10.1111/j.1742-1241.2008.01957.x.

31. Sarich PE, Ding D, Sitas F, Weber MF. Co-occurrence of chronic disease lifestyle risk factors in middle-aged and older immigrants: a cross-sectional analysis of 264,102 Australians. Prev Med. 2015;81:209-15. https://doi.org/10. 1016/j.ypmed.2015.09.004.

32. Barnett A, Cerin E, Zhang CJP, Sit CHP, Johnston JM, Cheung MMC, et al. Associations between the neighbourhood environment characteristics and physical activity in older adults with specific types of chronic conditions: the ALECS cross-sectional study. Int J Behav Nutr Phys Act. 2016;13:53. https:// doi.org/10.1186/s12966-016-0377-7.

33. Cerin E, Barnett A, Sit CH, Cheung MC, Lee LC, Ho SY, et al. Measuring walking within and outside the neighborhood in Chinese elders: reliability and validity. BMC Public Health. 2011;11:851. https://doi.org/10.1186/1471-2458-11-851.

34. Cerin E, Zhang CJ, Barnett A, Sit CH, Cheung MM, Johnston JM, et al. Associations of objectively-assessed neighborhood characteristics with older adults' total physical activity and sedentary time in an ultra-dense urban environment: findings from the ALECS study. Health Place. 2016:42:1-10. https://doi.org/10.1016/j.healthplace.2016.08.009.

35. Nathan A, Wood L, Giles-Corti B. Exploring socioecological correlates of active living in retirement village residents. J Aging Phys Act. 2014;22:1-15. https://doi.org/10.1123/japa.2012-0189.

36. Boakye-Dankwa E, Barnett A, Pachana NA, Turrell G, Cerin E. Associations between latent classes of perceived neighborhood destination accessibility and walking behaviors in older adults of a low-density and a high-Density City. J Aging Phys Act. 2019:1-12. https://doi.org/10.1123/japa.2018-0297.

37. Da WW, Garcia A. Later life migration: sociocultural adaptation and changes in quality of life at settlement among recent older Chinese immigrants in Canada. Act Adapt Aging. 2015;39(3):214-42. https://doi.org/10.1080/ 01924788.2015.1063330.

38. Ip D, Lui CW, Chui WH. Veiled entrapment: a study of social isolation of older Chinese migrants in Brisbane, Queensland. Aging Soc. 2007;27:719-38. https://doi.org/10.1017/S0144686X07006083.

39. Wong ST, Yoo GJ, Stewart AL. Examining the types of social support and actual sources of support in older Chinese and Korean immigrants. Int J Aging Human Development. 2005;61(2):105-21. https://doi.org/10.2190/ AJ62-QQKT-YJ47-B1T8.

40. Cerin E, Leslie E, Sugiyama T, Owen N. Perceived barriers to leisure-time physical activity in adults: an ecological perspective. J Phys Act Health. 2010; 7(4):451-9.

41. Dall PM, Ellis SLH, Ellis BM, Grant PM, Colyer A, Gee NR, et al. The influence of dog ownership on objective measures of free-living physical activity and sedentary behaviour in community-dwelling older adults: a longitudinal case-controlled study. BMC Public Health. 2017;17(1):496. https://doi.org/10. 1186/s12889-017-4422-5.
42. Yabroff KR, Troiano RP, Berrigan D. Walking the dog: is pet ownership associated with physical activity in California? J Phys Act Health. 2008;5(2): 216-28.

43. Wong R. International migration in older age: migration and socioeconomic conditions in the older age groups. Demos. 2001;14:16-7.

44. Lai DWL. Cultural factors and preferred living arrangement of aging Chinese Canadians. J Hous Elderly. 2005;19(2):71-86. https://doi.org/10.1300/ J081v19n02_05.

45. Luo H. Strengthening social capital through residential environment development for older Chinese in a Canadian context. J Gerontol Soc Work. 2016;59(1):16-34. https://doi.org/10.1080/01634372.2015.1118716.

46. Dong XQ, Chang ES, Wong E, Simon M. Perceptions and negative effect of loneliness in a Chicago Chinese population of older adults. Arch Gerontol Geriatr. 2012;54:151-9. https://doi.org/10.1016/j.archger.2011.04.022.

47. Osypuk TL, Diez Roux AV, Hadley C, Kandula NR. Are immigrant enclaves healthy places to live? The multi-ethnic study of atherosclerosis. Soc Sci Med. 2009;69(1):110-20. https://doi.org/10.1016/j.socscimed.2009.04.010.

48. Gilbert PA, Khokhar S. Changing dietary habits of ethnic groups in Europe and implications for health. Nutr Rev. 2008;66(4):203-15. https://doi.org/10. 1111/j.1753-4887.2008.00025.x.

49. Liu X. International perspectives on food safety and regulations - a need for harmonized regulations: perspectives in China. J Sci Food Agric. 2014;94(10): 1928-31. https://doi.org/10.1002/jsfa.6646.

50. Alcorn T, Ouyang Y. China's invisible burden of foodborne illness. Lancet. 2012;379(9818):789-90. https://doi.org/10.1016/s0140-6736(12)60330-4.

51. World Health Organization. WHO estimates of the global burden of foodborne diseases: foodborne disease burden epidemiology reference group 2007-2015. Geneva: WHO; 2015.

52. Jih J, Le G, Woo K, Tsoh JY, Stewart S, Gildengorin G, et al. Educational interventions to promote healthy nutrition and physical activity among older Chinese Americans: a cluster-randomized trial. Am J Public Health. 2016;106(6):1092-8. https://doi.org/10.2105/AJPH.2016.303111.

53. Rosenmöller DL, Gasevic D, Seidell J, Lear SA. Determinants of changes in dietary patterns among Chinese immigrants: a cross-sectional analysis. Int J Behav Nutr Phys Act. 2011;8:42. https://doi.org/10.1186/1479-5868-8-42.

54. Satia-Abouta J, Patterson RE, Kristal AR, Teh C, Tu SP. Psychosocial predictors of diet and acculturation in Chinese American and Chinese Canadian women. Ethn Health. 2002:7(1):21-39.

55. Guillaumie L, Godin G, Vézina-Im LA. Psychosocial determinants of fruit and vegetable intake in adult population: a systematic review. Int J Behav Nutr Phys Act. 2010;7:12. https://doi.org/10.1186/1479-5868-7-12.

56. Kushida O, Iriyama Y, Murayama N, Saito T, Yoshita K. Associations of selfefficacy, social support, and knowledge with fruit and vegetable consumption in Japanese workers. Asia Pac J Clin Nutr. 2017;26(4):725-30. https://doi.org/10.6133/apjen.062016.06.

57. Petroka K, Campbell-Bussiere R, Dychtwald DK, Milliron BJ. Barriers and facilitators to healthy eating and disease self-management among older adults residing in subsidized housing. Nutr Health. 2017;23(3):167-75. https://doi.org/10.1177/0260106017722724.

58. Pitt E, Gallegos D, Comans T, Cameron C, Thornton L. Exploring the influence of local food environments on food behaviours: a systematic review of qualitative literature. Public Health Nutr. 2017;20(13):2393-405 https://doi.org/10.1017/S1368980017001069.

59. Inglis V, Ball K, Crawford D. Why do women of low socioeconomic status have poorer dietary behaviours than women of higher socioeconomic status? A qualitative exploration. Appetite. 2005;45(3):334-43. https://doi. org/10.1016/j.appet.2005.05.003.

60. Rawlins E, Baker G, Maynard M, Harding S. Perceptions of healthy eating and physical activity in an ethnically diverse sample of young children and their parents: the DEAL prevention of obesity study. J Hum Nutr Diet. 2013; 26(2):132-44. https://doi.org/10.1111/j.1365-277X.2012.01280.x.

61. Welin L, Tibblin G, Svärdsudd K, Tibblin B, Ander-Peciva S, Larsson B, et al. Prospective study of social influences on mortality. The study of men born in 1913 and 1923. Lancet. 1985;1(8434):915-8. https://doi.org/10.1016/s01406736(85)91684-8.

62. Kawachi I, Colditz GA, Ascherio A, Rimm EB, Giovannucci E, Stampfer MJ, et al. A prospective study of social networks in relation to total mortality and cardiovascular disease in men in the USA. J Epidemiol Community Health. 1996:50(3):245-51. https://doi.org/10.1136/jech.50.3.245.

63. Cacioppo JT, Hawkley LC, Berntson GG, Ernst JM, Gibbs AC, Stickgold R, et al. Do lonely days invade the nights? Potential social modulation of sleep 
efficiency. Psychol Sci. 2002;13(4):384-7. https://doi.org/10.1111/1467-9280 00469.

64. Wilson RS, Krueger KR, Arnold SE, Schneider JA, Kelly JF, Barnes LL, et al. Loneliness and risk of Alzheimer disease. Arch Gen Psychiatry. 2007;64(2): 234-40. https://doi.org/10.1001/archpsyc.64.2.234.

65. Cacioppo JT, Hughes ME, Waite LJ, Hawkley LC, Thisted RA. Loneliness as a specific risk factor for depressive symptoms: cross-sectional and longitudinal analyses. Psychol Aging. 2006;21(1):140-51. https://doi.org/10.1037/08827974.21.1.140.

66. Lai DW, Chau SB. Effects of service barriers on health status of older Chinese immigrants in Canada. Soc Work. 2007;52(3):261-9.

67. Veninga J. Social capital and health: maximizing the benefits. Health Policy Research Bulletin. 2006;12:21-4

68. Makwarinma E, Stewart M, Jones Z, Makumbe K, Shizha E, Spitzer D. Senior immigrants' support needs and preferences of support intervention programs. In: Durst D, MacLean M, editors. Diversity and aging among immigrant seniors in Canada: changing faces and greying temples. Calgary: Detselig Enterprises Ltd.; 2010.

69. Wong ST, Yoo GJ, Stewart AL. An empirical evaluation of social support and psychological well-being in older Chinese and Korean immigrants. Ethn Health. 2007;12(1):43-67. https://doi.org/10.1080/13557850600824104.

70. Adams MA, Frank LD, Schipperijn J, Smith G, Chapman J, Christiansen LB, et al. International variation in neighborhood walkability, transit, and recreation environments using geographic information systems: the IPEN adult study. Int J Health Geogr. 2014;13:43. https://doi.org/10.1186/1476072X-13-43.

71. Cerin E, Cain KL, Conway TL, Van Dyck D, Hinckson E, Schipperijn J, et al. Neighborhood environments and objectively measured physical activity in 11 countries. Med Sci Sports Exerc. 2014;46(12):2253-64. https://doi.org/10. 1249/MSS.0000000000000367.

72. Taylor VM, Cripe SM, Acorda E, The C, Coronado G, Do H, et al. Development of an ESL curriculum to educate Chinese immigrants about physical activity. J Immigr Minor Health. 2008;10(4):379-87. https://doi.org/ 10.1007/s10903-007-9085-3

73. Giles-Corti B, Sallis JF, Sugiyama T, Frank LD, Lowe M, Owen N. Translating active living research into policy and practice: one important pathway to chronic disease prevention. J Public Health Policy. 2015;36:231-4. https:// doi.org/10.1057/jphp.2014.53.

\section{Publisher's Note}

Springer Nature remains neutral with regard to jurisdictional claims in published maps and institutional affiliations.

Ready to submit your research? Choose BMC and benefit from:

- fast, convenient online submission

- thorough peer review by experienced researchers in your field

- rapid publication on acceptance

- support for research data, including large and complex data types

- gold Open Access which fosters wider collaboration and increased citations

- maximum visibility for your research: over $100 \mathrm{M}$ website views per year

At BMC, research is always in progress.

Learn more biomedcentral.com/submissions 